\title{
Optimizing EELS data sets using 'a priori’ spectrum simulation
}

\author{
Nanda K Menon and John A Hunt \\ Gatan, Inc., 5933 Coronado Lane, Pleasanton, \\ CA 94588 USA
}

Electron energy-loss spectroscopy (EELS) is widely recognised as a powerful microanalytical technique applicable to a range of materials. The detection and reliable quantification of EELS core edges for an element in a given sample can be strongly influenced by parameters such as acquisition time, collection angle and the choice of background and signal windows. Selecting the optimal values for these quantities has thus far proceeded largely on a trial-and-error basis.

In view of this, a software package has been developed that calculates optimized conditions for acquiring and processing EEL spectra given some a priori knowledge of the specimen. The software first simulates a complete spectrum for an arbitrary, user-specified sample including the effect of shot noise [1]. The spectrum synthesis uses as default the Ritchie-Howie model [2] to simulate the low-loss and HartreeSlater cross-sections [3] to compute the core level scattering, although user-specified cross-sections may also be incorporated. An example of their contributions to the overall EEL scattering is given in Figure 1, which considers the case of $\mathrm{Al}_{2} \mathrm{O}_{3}$. The calculations are in reasonable agreement with experiment in many cases as illustrated in Figure 2, which shows a comparison between the calculated and experimental spectrum for $\mathrm{Al}_{2} \mathrm{O}_{3}$ for a primary energy of $200 \mathrm{keV}$ and a collection angle of $100 \mathrm{mrad}$.

The software is written for the DigitalMicrograph ( ( $)$ Gatan, Inc. ) software environment and uses its scripting language for configuring the user interface. Figure 3 illustrates the user interface in which a] shows the periodic table-like specification of sample information and b] the interface for the calculations, which are conducted by invoking $\mathrm{C}++$ functions for improved execution speed. Several spectra can then be computed per second.

In addition to spectrum calculation, the software contains optimization routines for EELS quantification. Firstly, the signal of interest is extracted by fitting and extrapolating a power-law background. Then, a mutidimensional simplex algorithm [4] explores the variation of the fractional difference between the true and extracted counts with parameters including beam dose, collection angle and background and signal positions /widths. In addition, users are given the capability to choose the fixed and variable parameters. Figure 3. demonstrates the result of the optimization ( which takes about 15 seconds on $800 \mathrm{Mhz}$ PC ) as applied to the quantification of trace concentrations of Ca embedded in a $10 \mathrm{~nm}$ Carbon matrix at the Ca L-edge. In conjunction with locating the optimal values, an estimate of the Minimum Detectable Mass ( MDM ) and Minimum Detectable Number of atoms ( MDN ) is given on output. In this case, single atom detection is predicted for an electron dose of about $1.0 \times 10^{8} \mathrm{Cm}^{-2}$ (which, for a probe size of $1 \mathrm{~nm}$ and $0.5 \mathrm{nA}$ of current corresponds to an acquisition time of at least $\sim 0.5 \mathrm{~s}$ ). The results compare favorably with the experimental observations of Leapman and Rizzo [5] for $\mathrm{Ca}$ quantification in a thin $\mathrm{C}$ matrix. These methods have also been extended to include elemental and ratio mapping. For user-specified edges, the software outputs optimal values for the signal and background positions and widths. As an illustration, Figure 4. gives the result at the V L edge for a $20 \mathrm{~nm}$ sample of $\mathrm{TiO}_{2}$ with $20 \% \mathrm{~V}$ present. The figure demonstrates the usefulness of the software in determining the optimal window parameters in the case of overlapping core edges.

In conclusion, the EELS simulation and optimization software package is envisaged to be a significant aid for EELS experimentalists to decide a priori, for a given sample and acquisition conditions, if a given EELS / EFTEM experiment is worth performing. Furthermore, it provides users with estimates for the optimal acquisition and processing parameters for elemental quantification at a given edge. The methods can be readily extended to compute and acquire optimized elemental /ratio maps using the conventional three/two window methods.

\section{References}

1. N K Menon and O L Krivanek Synthesis of Electron Energy Loss Spectra for the Quantification of Detection Limits ( submitted to Microscopy and Microanalysis )

2. R H Ritchie and A Howie Electron excitation and the optical potential in electron microscopy. Phil Mag 36 (1977):463 
3. P Rez Cross-sections for energy-loss spectroscopy. Ultramicroscopy 9 (1982):283

4. WH Press et al. Numerical Recipes in C. $2^{\text {nd }}$ Edition (1992) Cambridge University Press.

5. RD Leapman RD, NW Rizzo (1999) Towards single atom analysis of biological structures.

Ultramicroscopy 78:251

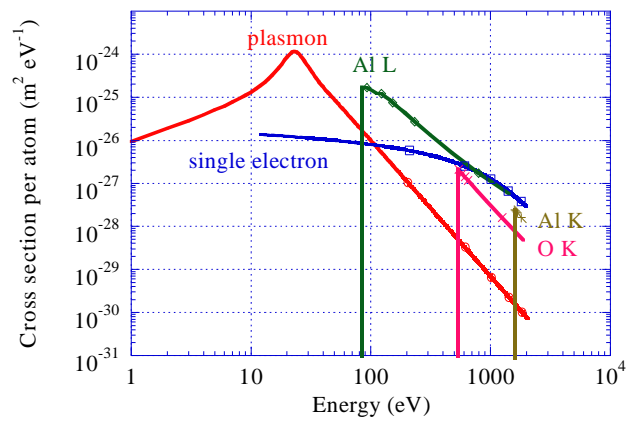

Figure 1. Contributions to the EEL spectrum of $\mathrm{Al}_{2} \mathrm{O}_{3}$

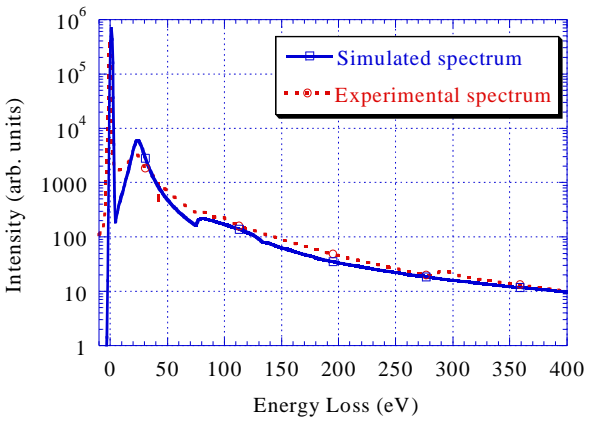

Figure 2. Comparison between simulated and experimental spectrum for $\mathrm{Al}_{2} \mathrm{O}_{3}$ at $200 \mathrm{keV}$ and $100 \mathrm{mrad}$ collection angle.
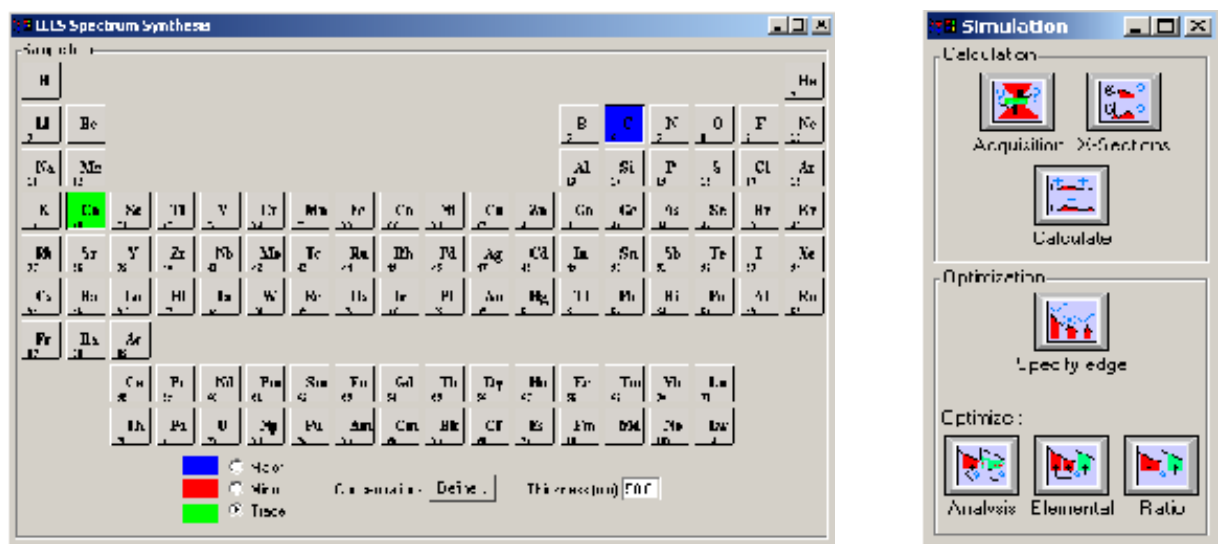

Figure 3. User interface for element specification and simulation/optimization

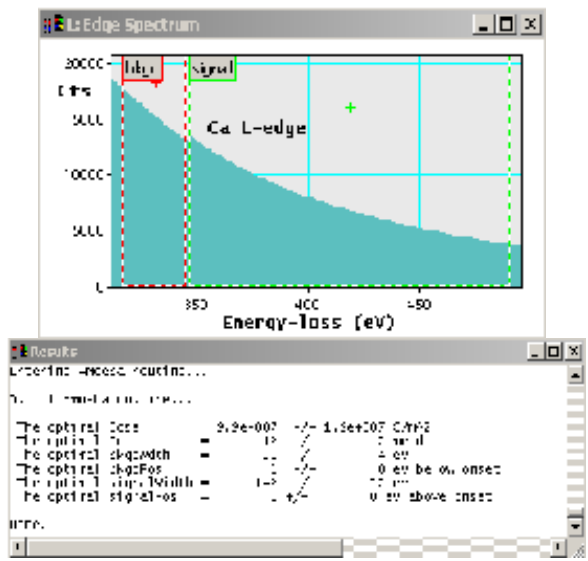

Figure 4. Quantification of $1 \% \mathrm{Ca}$ in a $10 \mathrm{~nm}$ $\mathrm{C}$ matrix at Ca L-edge. The display is updated as the optimization progresses, with final values output to the Results window.

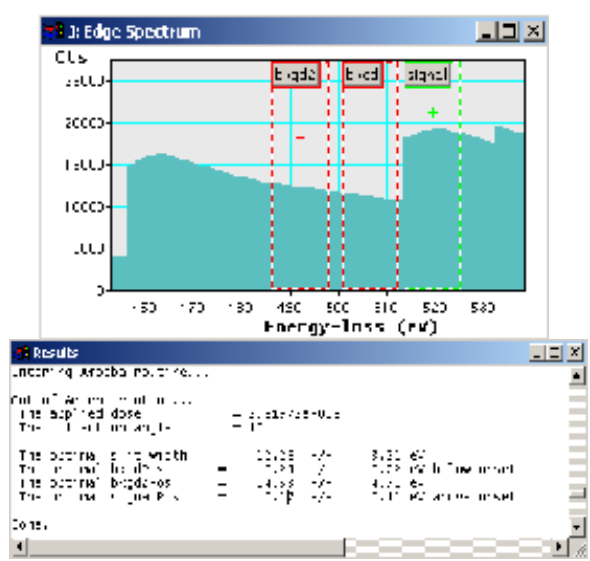

Figure 5. Optimizing the 3-window elemental mapping for $20 \% \mathrm{~V}$ in a $20 \mathrm{~nm}$ thick $\mathrm{TiO}_{2}$ film. 